\title{
Ultrafast Synthesis of Multifunctional N-Doped Graphene Foam in an Ethanol Flame
}

\author{
Xusheng Du, Hong-Yuan Liu, Yiu-Wing Mai \\ Centre for Advanced Materials Technology (CAMT), School of Aerospace Mechanical \& Mechatronic \\ Engineering J07, The University of Sydney, Sydney, NSW 2006, Australia
}


Table S1. Fabrication method of some typical graphene foams and their absorption capacities

\begin{tabular}{|c|c|c|c|c|c|c|}
\hline $\begin{array}{l}\text { Foam } \\
\text { material }\end{array}$ & $\begin{array}{l}\text { Fabrication } \\
\text { method }\end{array}$ & $\begin{array}{l}\text { Synthesis } \\
\text { time }\end{array}$ & $\begin{array}{l}\text { Density } \\
\left(\mathrm{mg} / \mathrm{cm}^{3}\right)\end{array}$ & $\begin{array}{l}\text { Geometry } \\
\text { controllability }\end{array}$ & $\begin{array}{l}\text { Absorption } \\
\text { capacity }(\mathrm{g} / \mathrm{g})\end{array}$ & $\operatorname{Ref} \#$ \\
\hline $\begin{array}{l}\text { N-doped } \\
\text { graphene } \\
\text { foam }\end{array}$ & $\begin{array}{l}\text { Polymer foam } \\
\text { template and } \\
\text { ethanol flame }\end{array}$ & $\begin{array}{l}\text { Short } \\
\text { (minutes) }\end{array}$ & 2.7 & $\begin{array}{l}\text { Directly depend on } \\
\text { polymer foam } \\
\text { template }\end{array}$ & $\begin{array}{l}\text { 241(hexane); } \\
\text { 316(toluene) }\end{array}$ & $\begin{array}{l}\text { Present } \\
\text { work }\end{array}$ \\
\hline $\begin{array}{l}\text { N-doped } \\
\text { graphene } \\
\text { foam }\end{array}$ & $\begin{array}{l}\text { Hydrothermal } \\
\text { with pyrrole in } \\
\text { autoclave; } \\
\text { freeze-drying } \\
\text { and thermal } \\
\text { annealing at } \\
1050^{\circ} \mathrm{C} \text { in } \mathrm{Ar}\end{array}$ & $\begin{array}{l}\text { Long } \\
\text { (hours) }\end{array}$ & 2.1 & $\begin{array}{l}\text { Depend on shape of } \\
\text { reaction vessel }\end{array}$ & $\sim 200$ (toluene) & 5 \\
\hline $\begin{array}{l}\text { Graphene } \\
\text { foam }\end{array}$ & $\begin{array}{l}\text { Filtration and } \\
\text { chemical } \\
\text { reaction with } \\
\text { hydrazine in } \\
\text { autoclave }\end{array}$ & $\begin{array}{l}\text { Long } \\
\text { (hours) }\end{array}$ & 30 & $\begin{array}{l}\text { Depend on filtration } \\
\text { and hydrazine } \\
\text { reduction process } \\
\text { usually paper form }\end{array}$ & $\begin{array}{l}\sim 36 \text { (hexane); } \\
\sim 18 \text { (toluene) }\end{array}$ & 38 \\
\hline $\begin{array}{l}\text { Graphene } \\
\text { foam }\end{array}$ & $\begin{array}{l}\text { Freeze-drying } \\
\text { and thermal } \\
\text { treatment at } \\
1000-2400^{\circ} \mathrm{C} \\
\text { under high } \\
\text { vacuum }\end{array}$ & $\begin{array}{l}\text { Long } \\
\text { (hours) }\end{array}$ & 4.3 & $\begin{array}{l}\text { Depend on shape of } \\
\text { drying vessel }\end{array}$ & 113(toluene) & 2 \\
\hline $\begin{array}{l}\text { Graphene } \\
\text { foam }\end{array}$ & $\begin{array}{l}\text { Hydrothermal } \\
\text { reaction with } \\
\text { ammonia in } \\
\text { autoclave and } \\
\text { freeze-drying }\end{array}$ & $\begin{array}{l}\text { Long } \\
\text { (hours) }\end{array}$ & 12 & $\begin{array}{l}\text { Depend on shape of } \\
\text { the reaction vessel }\end{array}$ & $\begin{array}{l}44 \text { (hexane) } \\
54.8 \text { (toluene) }\end{array}$ & 39 \\
\hline $\begin{array}{l}\text { Graphene } \\
\text { foam }\end{array}$ & $\begin{array}{l}\text { Hydrothermal } \\
\text { reaction with } \\
\text { thiourea in } \\
\text { autoclave and } \\
\text { freeze-drying }\end{array}$ & $\begin{array}{l}\text { Long } \\
\text { (hours) }\end{array}$ & - & $\begin{array}{l}\text { Depend on shape of } \\
\text { reaction vessel }\end{array}$ & 75(hexane) & 40 \\
\hline $\begin{array}{l}\text { Graphene } \\
\text { foam }\end{array}$ & $\begin{array}{l}\text { Freeze-drying in } \\
\text { presence of } \\
\text { surfactantand } \\
\text { thermal } \\
\text { annealing in } \mathrm{Ar}\end{array}$ & $\begin{array}{l}\text { Long } \\
\text { (hours) }\end{array}$ & 2 & $\begin{array}{l}\text { Depend on shape of } \\
\text { drying vessel }\end{array}$ & 125(hexane) & 42 \\
\hline $\begin{array}{l}\text { Graphene/ } \\
\text { CNT foam }\end{array}$ & $\begin{array}{l}\text { Freeze-drying } \\
\text { and reduction } \\
\text { with hydrazine } \\
\text { vapor }\end{array}$ & $\begin{array}{l}\text { Long } \\
\text { (hours) }\end{array}$ & 1.4 & $\begin{array}{l}\text { Depend on shape of } \\
\text { freeze-drying vessel }\end{array}$ & 215(hexane) & 8 \\
\hline
\end{tabular}


Table S2. Fabrication method of some graphene foams, their density and compression properties

\begin{tabular}{|c|c|c|c|c|c|}
\hline $\begin{array}{l}\text { Foam } \\
\text { material }\end{array}$ & Fabrication method & $\begin{array}{l}\text { Density } \\
\left.(\mathrm{mg} \mathrm{cm})^{-3}\right)\end{array}$ & $\begin{array}{l}\text { Elastic } \\
\text { modulus } \\
(\mathrm{kPa})\end{array}$ & $\begin{array}{l}\text { Maximum } \\
\text { compressive } \\
\text { stress at given } \\
\text { strain }(\mathrm{kPa}) \\
\end{array}$ & Ref \# \\
\hline $\begin{array}{l}\text { N-doped } \\
\text { graphene foam }\end{array}$ & $\begin{array}{l}\text { Polymer foam template and } \\
\text { ethanol flame }\end{array}$ & $\begin{array}{l}2.7 \\
4.8 \\
10\end{array}$ & $\begin{array}{l}1.4 \\
2.1 \\
30\end{array}$ & $\begin{array}{l}0.3(50 \%) \\
1.9(50 \%) \\
2.3(50 \%)\end{array}$ & Present work \\
\hline $\begin{array}{l}\text { N-doped } \\
\text { graphene foam }\end{array}$ & $\begin{array}{l}\text { Hydrothermal with pyrrole } \\
\text { in autoclave; freeze-drying } \\
\text { and thermal annealing at } \\
1050^{\circ} \mathrm{C} \text { in } \mathrm{Ar}\end{array}$ & 2.1 & - & $2.5(50 \%)$ & 5 \\
\hline Graphene foam & $\begin{array}{l}\text { PU template thermal } \\
\text { annealing at } 1000^{\circ} \mathrm{C} \text { in } \mathrm{N}_{2}\end{array}$ & 4.7 & 250 & $8.9(34 \%)$ & 25 \\
\hline $\begin{array}{l}\text { Graphene } \\
\text { aerogel }\end{array}$ & $\begin{array}{l}\text { Hydrothermal reaction and } \\
\text { freeze-drying }\end{array}$ & 6.73 & 16.83 & $14.05(50 \%)$ & 15 \\
\hline Graphene foam & $\begin{array}{l}\text { Freeze-drying and thermal } \\
\text { treatment at } 1000-2400^{\circ} \mathrm{C} \\
\text { under high vacuum }\end{array}$ & 6.1 & $\sim 25$ & $\sim 6.5(50 \%)$ & 2 \\
\hline $\begin{array}{l}\text { Graphene } \\
\text { aerogel }\end{array}$ & $\begin{array}{l}\text { Hydrothermal reaction with } \\
\text { ammonia and paraphenylene } \\
\text { diamine and freeze drying }\end{array}$ & 4.5 & 20 & $8.5(50 \%)$ & 53 \\
\hline Graphene foam & $\begin{array}{l}\text { Hydrothermal reaction with } \\
\text { thiourea in autoclave and } \\
\text { freeze-drying }\end{array}$ & - & - & $14.0(25 \%)$ & 40 \\
\hline $\begin{array}{l}\text { Graphene/CNT } \\
\text { foam }\end{array}$ & $\begin{array}{l}\text { Freeze-drying and reduction } \\
\text { with hydrazine vapor }\end{array}$ & 5.6 & $>4$ & $6.2(50 \%)$ & 8 \\
\hline $\begin{array}{l}\text { Graphene } \\
\text { aerogel }\end{array}$ & $\begin{array}{l}\text { Hydrothermal reaction, } \\
\text { freeze-drying and microwave } \\
\text { annealing }\end{array}$ & $3-5$ & $\sim 16.7$ & $\sim 4.6(50 \%)$ & 9 \\
\hline $\begin{array}{l}\text { Graphene } \\
\text { cellular } \\
\text { monolith }\end{array}$ & $\begin{array}{l}\text { Hydrothermal reaction with } \\
\text { ascorbic acid followed by } \\
\text { freezing, thawing, reduction, } \\
\text { freeze-drying and thermal } \\
\text { annealing }\end{array}$ & 5.1 & 20 & $\sim 7.5(50 \%)$ & 14 \\
\hline
\end{tabular}



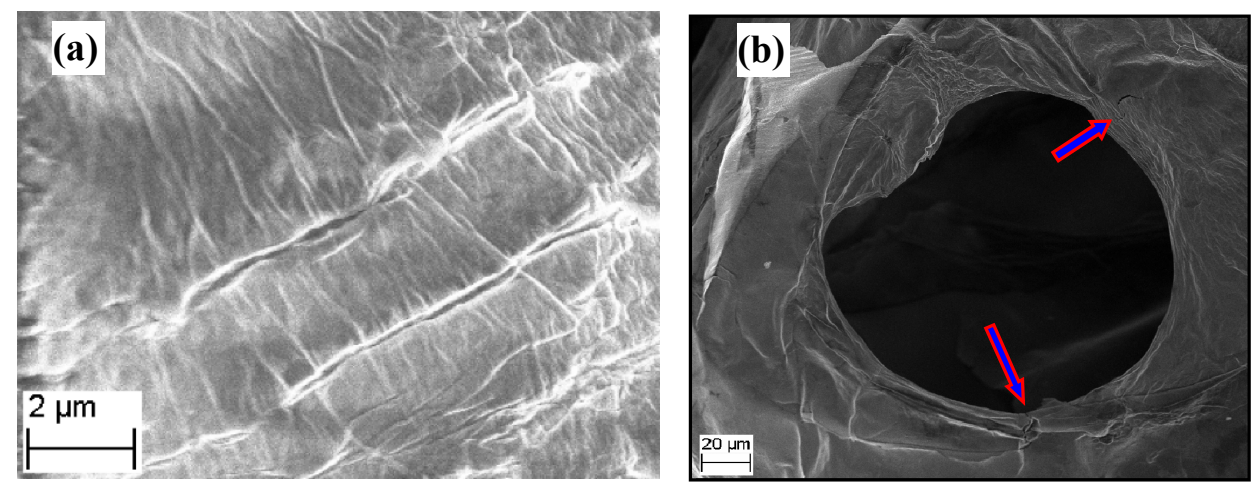

Figure S1. SEM images of (a) PU@GO foam and (b) fractured struts of NGF (micro-cracks are highlighted by arrows).
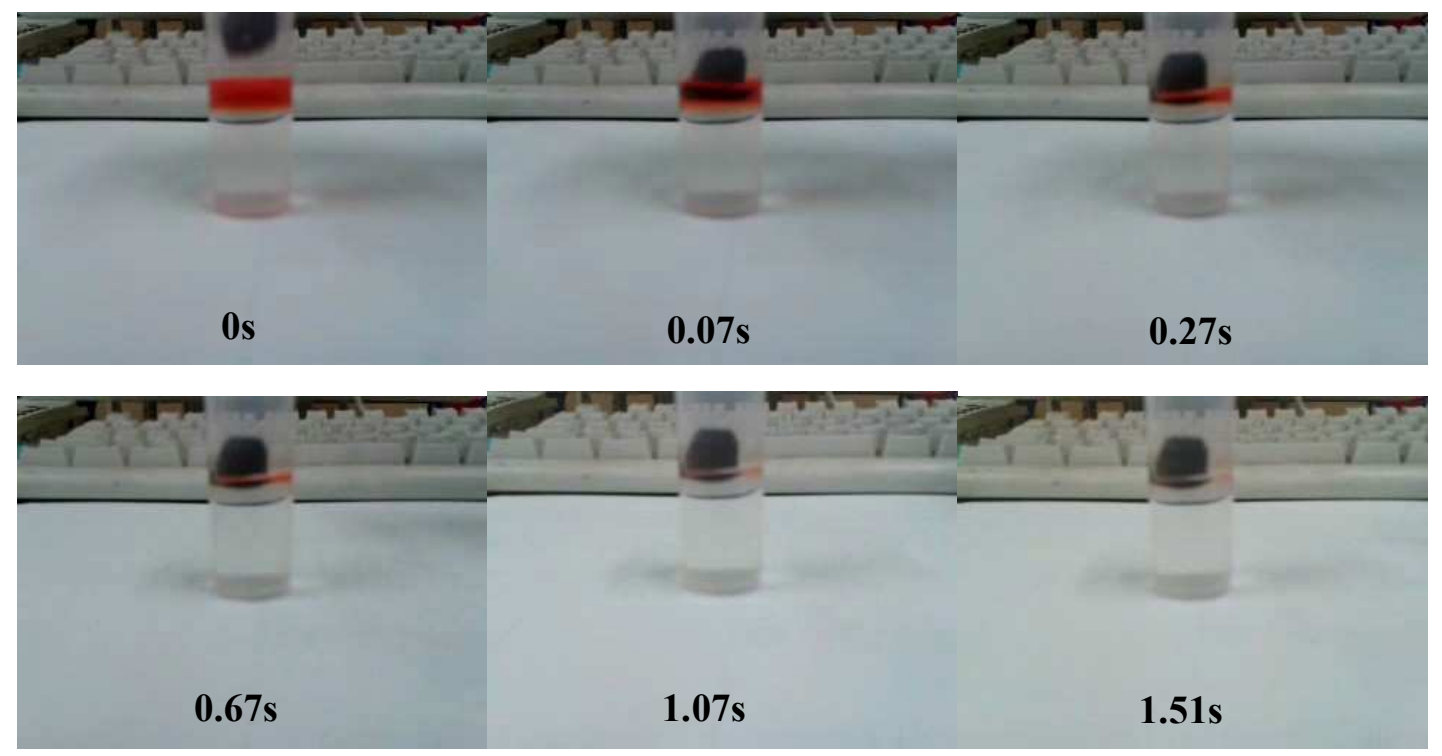

Figure S2. Digital images of absorption process of heptane (stained by Sudan III) on water by NGF within $<1.51 \mathrm{~s}$ taken at $0,0.07,0.27,0.67,1.07$, and $1.51 \mathrm{~s}$, respectively.

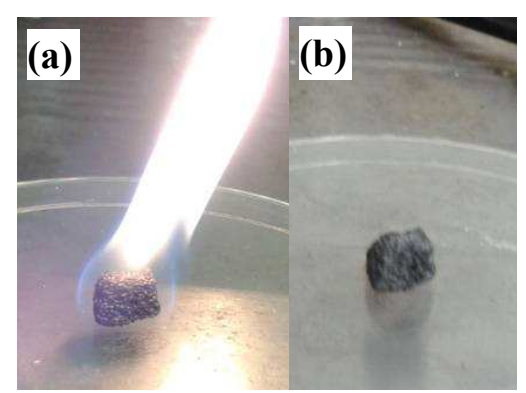

Figure S3. Digital image of NGF absorbed with heptane:

(a) during burning and (b) after combustion. 

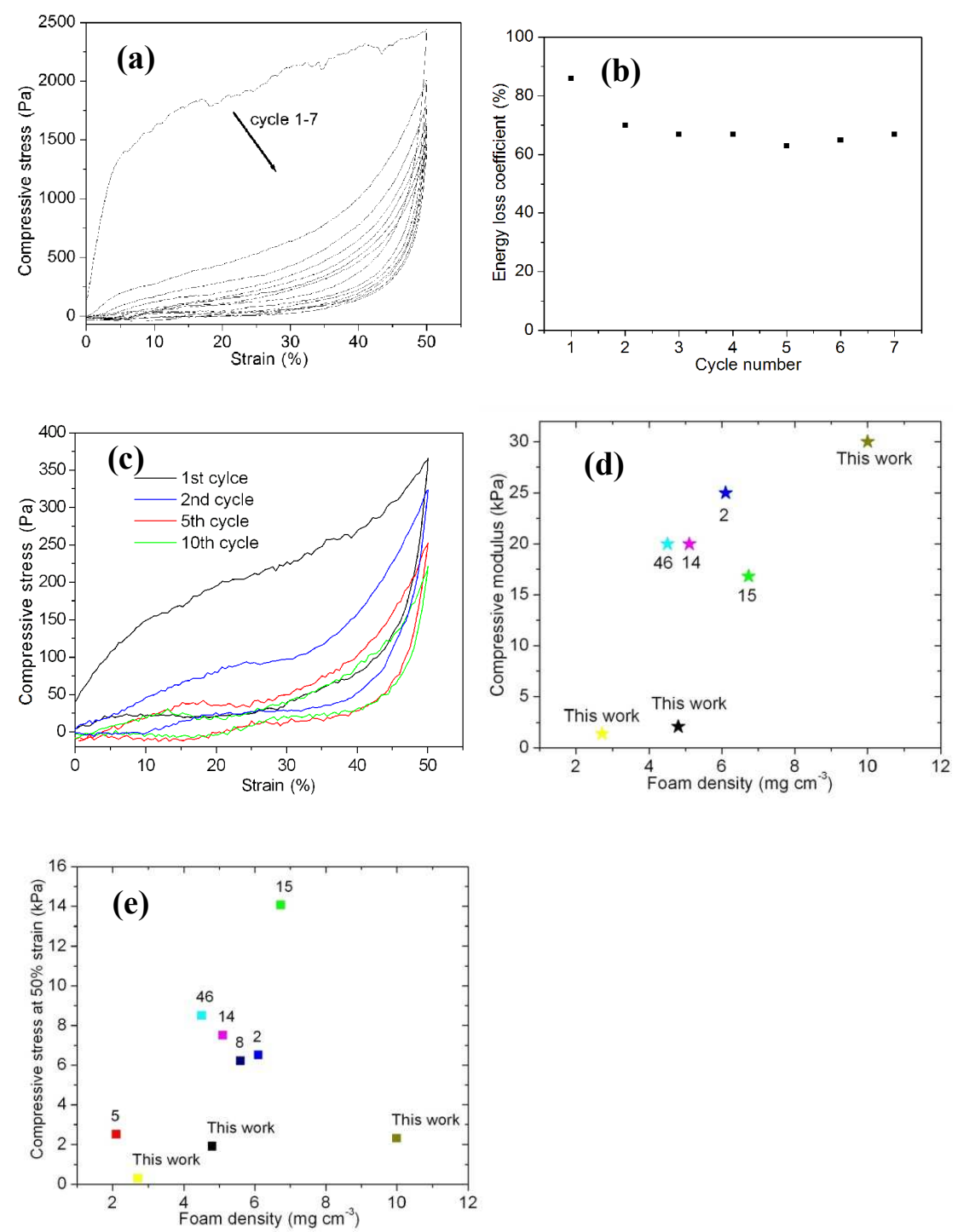

Figure S4. Multi-cycle compression stress-strain curves of NGF with (a) $10 \mathrm{mg} \mathrm{cm}^{-3}$ and (c) $2.7 \mathrm{mg} \mathrm{cm}^{-3}$. (b) History of energy loss coefficient for 7 compression cycles of NGF with $10 \mathrm{mg} \mathrm{cm}^{-3}$ at 50\% strain. (d) Compressive modulus and (e) compressive stress at 50\% strain of NGF in comparison with other reported graphene foams. Numbers beside the symbols are cited references.

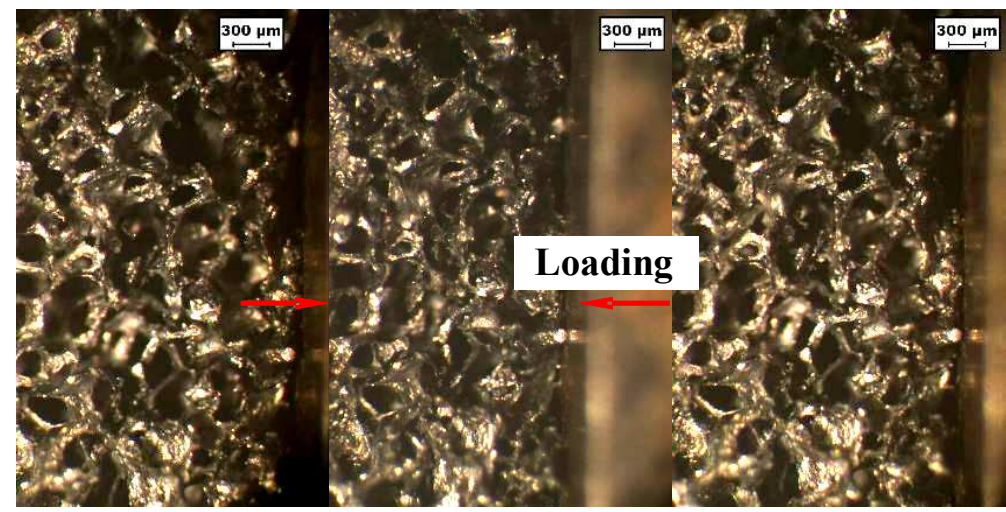

Fig S5. Optical microcopy images of NGF compressed to strains of 0 and $20 \%$, and its recovery. 


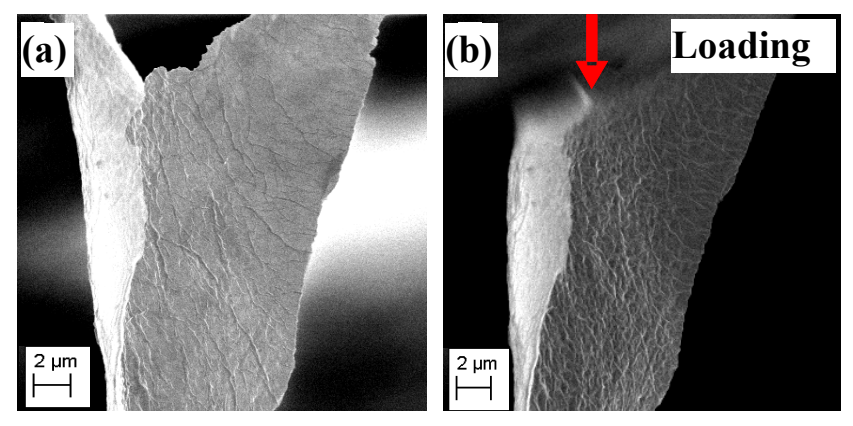

Figure S6. In-situ SEM images of graphenes on NGF: (a) no applied load, and (b) with applied compressive load.
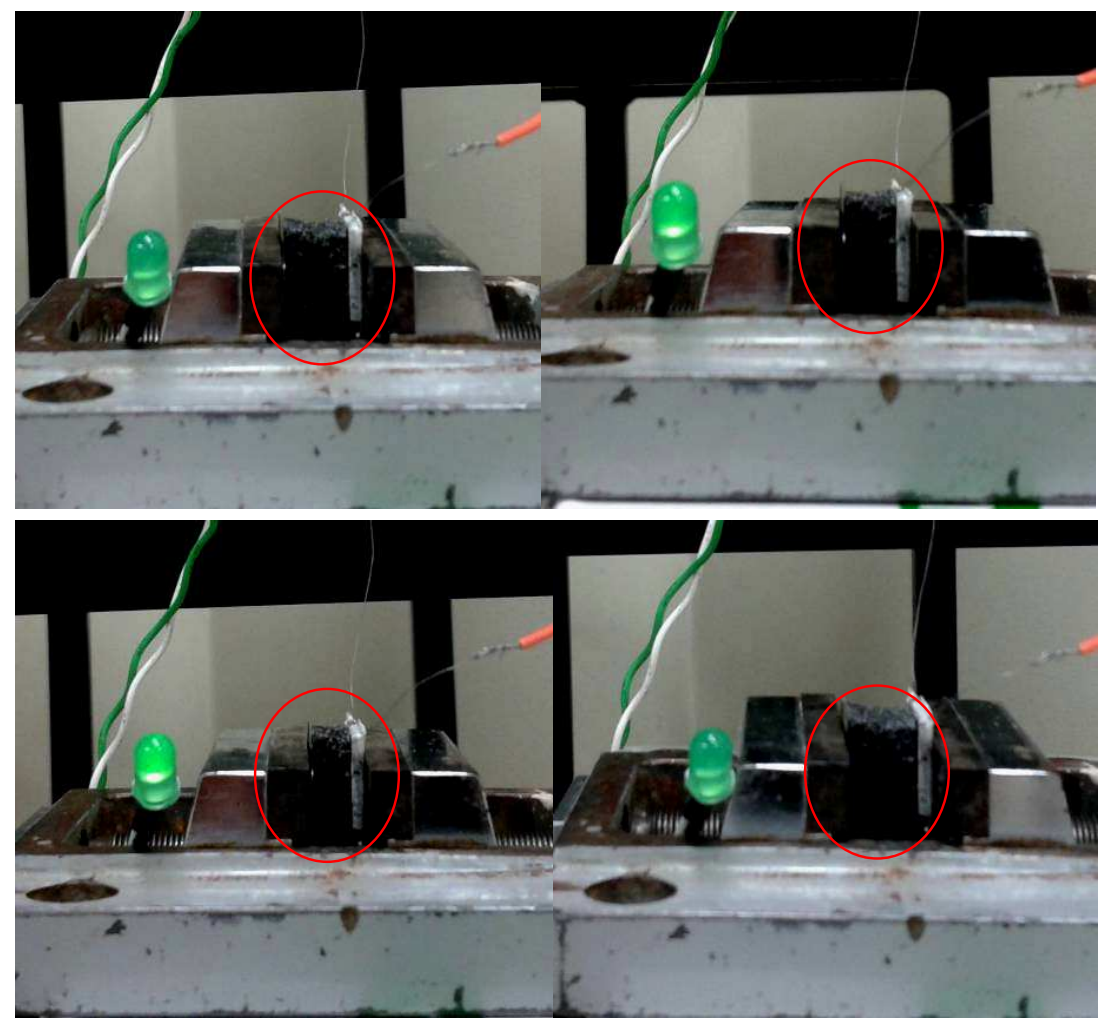

Figure S7. Electrical conductivity of NGF with applied compressive load and after release of load. 